\title{
Effects of Sensor Cover Damages on Point Clouds of Automotive Lidar
}

This paper was downloaded from TechRxiv (https://www.techrxiv.org).

\section{LICENSE}

CC BY 4.0

SUBMISSION DATE / POSTED DATE

$10-01-2022$ / 12-01-2022

CITATION

Schlager, Birgit; Goelles, Thomas; Watzenig, Daniel (2022): Effects of Sensor Cover Damages on Point Clouds of Automotive Lidar. TechRxiv. Preprint. https://doi.org/10.36227/techrxiv.18101279.v1

$\mathrm{DOI}$

10.36227/techrxiv.18101279.v1 


\section{Effects of Sensor Cover Damages on Point Clouds of Automotive Lidar}

\author{
$1^{\text {st }}$ Birgit Schlager \\ Virtual Vehicle Research GmbH and \\ Graz University of Technology \\ Graz, Austria \\ ORCID: 0000-0003-3290-5333
}

\author{
$2^{\text {nd }}$ Thomas Goelles \\ Virtual Vehicle Research GmbH \\ Graz, Austria \\ ORCID: 0000-0002-3925-6260
}

\author{
$3^{\text {rd }}$ Daniel Watzenig \\ Virtual Vehicle Research GmbH and \\ Graz University of Technology \\ Graz, Austria
}

ORCID: 0000-0002-5341-9708

\begin{abstract}
Safe automated driving requires reliable perception sensors with low fault rates. Detecting perception sensor faults before path planning avoids fault propagation through the processing pipeline of automated vehicles. As the basis for further development of fault detection algorithms, the present work presents effects of damaged lidar sensor covers considering scratches, cracks, and holes. We used an automotive lidar, which provides point clouds, and calculated deviations between the lidar points on a target and an ideal plane representing the target to evaluate the effect of damaged covers. Results show that sensor cover damages have an effect on point cloud data.

Index Terms - sensor fault, lidar, mechanical damage
\end{abstract}

\section{INTRODUCTION}

Automated driving is a promising technology for future mobility solutions, since automated vehicles are expected to enhance the safety of passengers by reducing the number of accidents and especially the number of fatal accidents [1], [2]. A main challenge is the step from "driver support features" to "automated driving features" since the responsibility is transferred from the human driver to the vehicle [3], [4]. Reliable perception sensors are an integral part of automated driving for transferring the responsibility to the vehicle.

In general, an automated vehicle works according to the sense-plan-act cycle [5]. In the sense phase the vehicle captures the environment and objects around the vehicle with perception sensors such as lidar, radar, and camera. Then the vehicle calculates its path between the captured objects in the plan phase and follows the calculated path in the act phase. Detecting sensor faults early in the sense-plan-act cycle avoids fault propagation through the processing pipeline and increases the safety of passengers.

Our review paper shows that several sensor fault classes are already well covered by the literature [6]. Especially, environmental effects in terms of weather conditions are mentioned in several publications [7]-[11]. Furthermore, the

The publication was written at Virtual Vehicle Research GmbH in Graz, Austria. The authors would like to acknowledge the financial support within the COMET K2 Competence Centers for Excellent Technologies from the Austrian Federal Ministry for Climate Action (BMK), the Austrian Federal Ministry for Digital and Economic Affairs (BMDW), the Province of Styria (Dept. 12) and the Styrian Business Promotion Agency (SFG). The Austrian Research Promotion Agency (FFG) has been authorised for the programme management. influence of sensor blockage is investigated in one work [12]. Damages of the sensor cover like scratches, cracks, and holes are discussed barely in current literature. Only one work was found that mentions damages of lidar sensor covers in form of micro scratches caused by road dirt [13]. Their results show that dirty lidar sensor covers lead to a decreased sensor performance because of a lower transmission of light through the sensor cover. The effect of micro scratches on sensor data was not analyzed in their work.

The present work aims to close this gap in research and investigates the effects of damages on lidar sensor data. For that purpose, point clouds of an automotive rotating lidar were analyzed.

\section{TEST SETUP}

The test setup consisting of a lidar sensor and a target is explained in this section. Furthermore, this section includes images of damaged lidar sensor covers and describes the target setup used for the measurements.

The test setup is shown in Fig. 1. A lidar sensor, the Ouster OS1-64 Gen1 (OS1-64) [14], was used for the measurements. The OS1-64 is a $360^{\circ}$ automotive rotating lidar sensor with 64 beams in vertical direction and a wavelength of $850 \mathrm{~nm}$. Only the strongest reflection of each beam is considered in the point cloud which is the output format of the OS1-64 shown in Fig. 2 We used Robot Operating System $\left(\operatorname{ROS}^{\mathrm{TM}}\right)$ [15] for data acquisition.

The target was a $46 \mathrm{~cm} \times 46 \mathrm{~cm}$ aluminum plate which served as the carrier for reflective foils. We investigated different reflective foils concerning their reflectance depending on the incidence angle shown in Fig. 3 . We chose the white retro-reflective foil since it reflects light back to the lidar sensor under a wide range of incidence angles. Therefore, the setup is not susceptible to slight deviations from an orthogonal orientation between the sensor and the target. The reflectance of the white retro-reflective target is shown by the green dashed graph in Fig. 3. Furthermore, the figure illustrates Lambertian targets with $10 \%, 50 \%$, and $95 \%$ reflectance as dotted graphs [16] and two white diffuse reflective foils as solid graphs.

Damages, i.e. scratches, cracks, and holes depicted in Fig. 4 


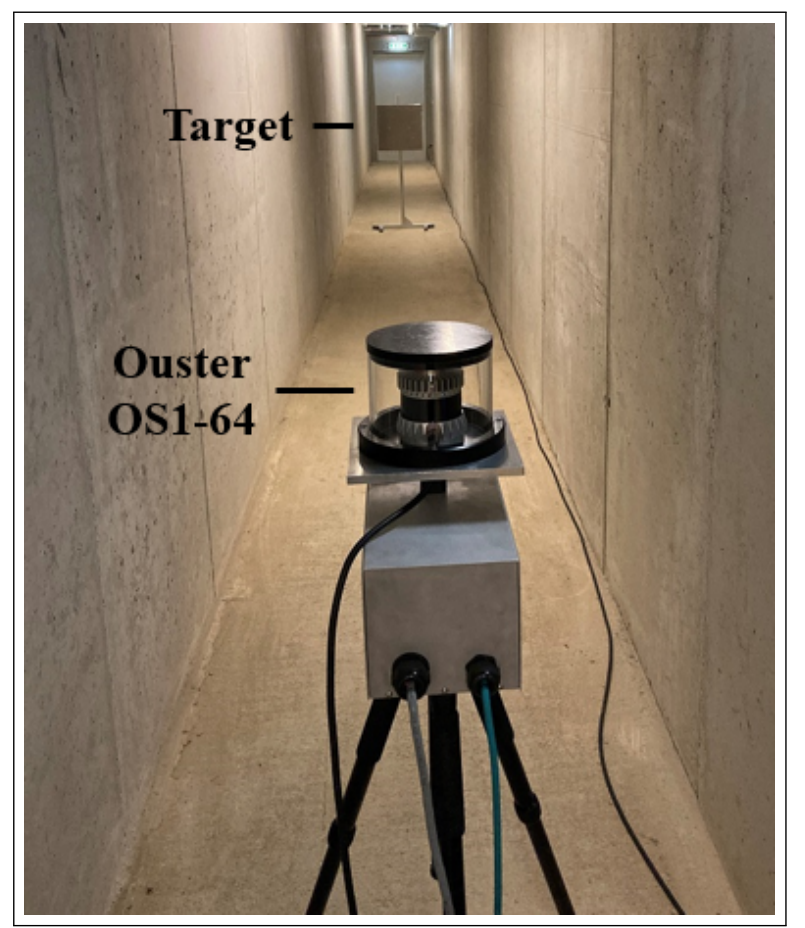

Fig. 1. Test setup including the OS1-64 and the aluminum target without any reflective foil attached. The target was placed in $9 \mathrm{~m}$ distance to the lidar sensor. The door behind the target was about $18 \mathrm{~m}$ away from the lidar sensor. Light was turned off during lidar measurements.

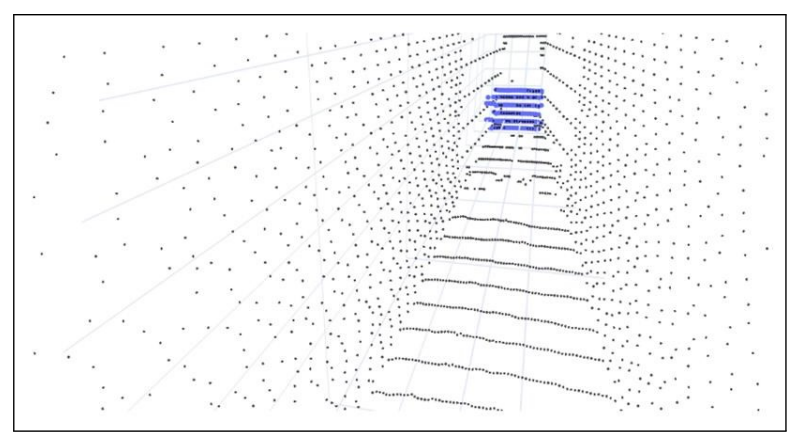

Fig. 2. Example point cloud of OS1-64. A retro-reflective target was used No additional plastic cover was applied (id:23).

were applied to the sensor covers of the OS1-64 to investigate its influence on sensor data. For that purpose, we added plastic covers of the type Plexiglas ${ }^{\circledR} \mathrm{XT}$ to the sensor to protect the original sensor cover. We used cylindrical plastic covers to cover the sensor's entire field of view of $360^{\circ}$ and labeled the sensor covers with cover IDs for the measurements. Throughout the present paper the cover IDs are written in brackets after the cover name.

\section{DATA ANALYSiS}

The OS1-64 shows effects on the measured range values if damages are applied. Therefore, we evaluated the effects of cover damages on point clouds of the OS1-64 by calculating the deviation from a ground truth target. An ideal plane represented by the plane equation served as the ground truth. The parameters of the plane equation $a x+b y+c z+d=0$

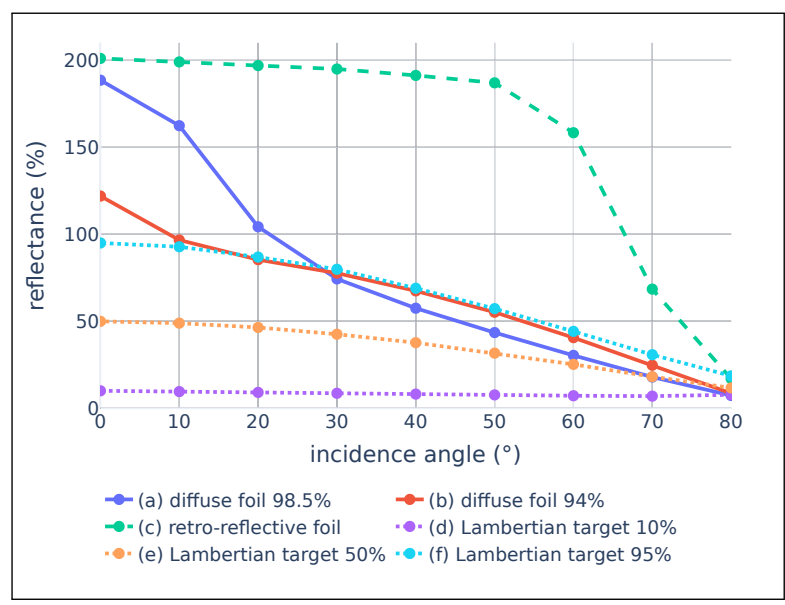

Fig. 3. Comparison of the reflectance of different targets depending on incidence angle. Following products were used: (a) Ingemann Components I-Reflect $0.23 \mathrm{~mm}\left[17\right.$, (b) $3 \mathrm{M}^{\mathrm{TM}}$ Scotchcal $^{\mathrm{TM}} 3635-100$ Light Enhancement Film White [18], and (c) $3 \mathrm{M}^{\mathrm{TM}}$ Engineer Grade ${ }^{\mathrm{TM}} \mathrm{DG}^{3}$ Prismatic Reflective Sheeting 3430 White [19]. The Lambertian targets of (d), (e), and (f) were adapted from [16] where Lambertian targets from Sphere Optics [20] were used. The reflectance was calibrated with Lambertian targets. Therefore, an ideal diffuse target with $100 \%$ reflectance results in a reflectance of $100 \%$ in the measurements. The reflectance can be higher than $100 \%$ in case that the target has specular reflection properties.

were derived via plane segmentation provided by the Open3D library [21]. The target, represented by blue dots in Fig. 2 , was isolated by limiting the volume to $-10 \mathrm{~m}<x<-9 \mathrm{~m}$, $-0.2 \mathrm{~m}<y<0.5 \mathrm{~m}$, and $-0.5 \mathrm{~m}<z<0.1 \mathrm{~m}$. Then the mean deviation of all points on the target to the ideal plane was calculated for 500 point clouds for each sensor cover. The Kernel Density Estimations (KDEs) of those 500 mean deviations per fault were calculated to show the effect of the applied faults.

\section{RESUlTS}

Fig. 5 illustrates a violin plot for each sensor cover. The deviations range between $0.023 \mathrm{~m}$ and $0.037 \mathrm{~m}$ in the case of no cover (id:23). The median of the reference cover (id:16) without any damages is at $0.0756 \mathrm{~m}$. All the other covers can be compared to this reference. The median deviation of the OS1-64 shifted from $0.029 \mathrm{~m}$ for no cover (id:23) to $0.0756 \mathrm{~m}$ for reference cover (id:16). Most of the faulty OS1-64 covers have deviations higher than the reference cover, except the cover with one crack. Except for multiple stone scratches (id:11) and diagonal stone scratches (id:17) the distributions are located outside of the range: mean $+/-1$ standard deviation of the reference. This range is illustrated by two vertical lines in Fig. 5. The deviation of the reference cover appears to be symmetrically distributed, while some damaged covers have skewed distributions.

\section{Discussion AND CONCLUSION}

The distributions of cracks and holes are located outside of the range of mean $+/-1$ standard deviation of the reference cover. Some of the distributions of scratches are located inside this range but the widths of those distributions deviate 


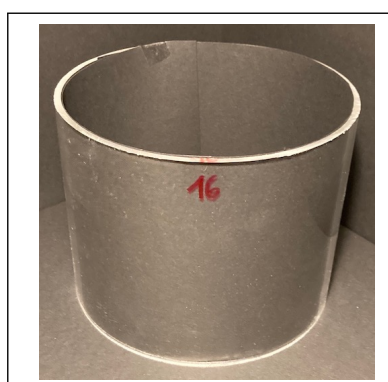

(a)

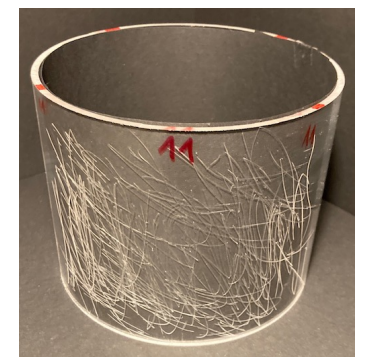

(c)

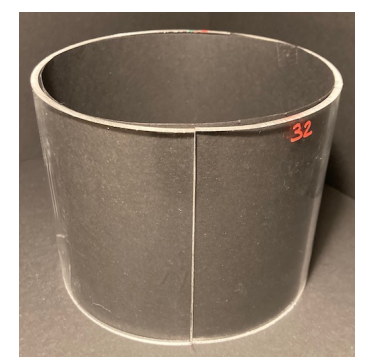

(e)

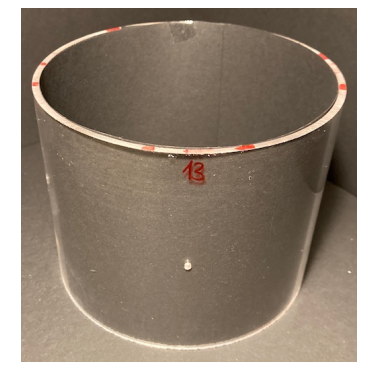

$(\mathrm{g})$

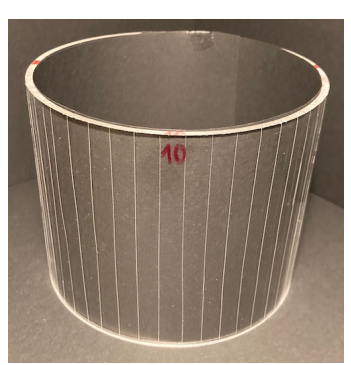

(b)

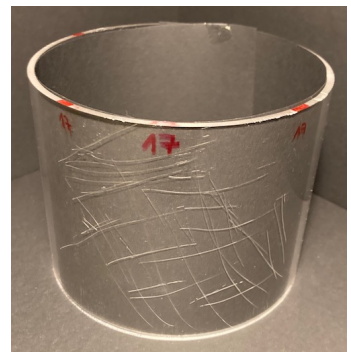

(d)

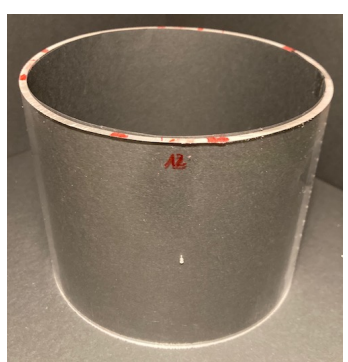

(f)

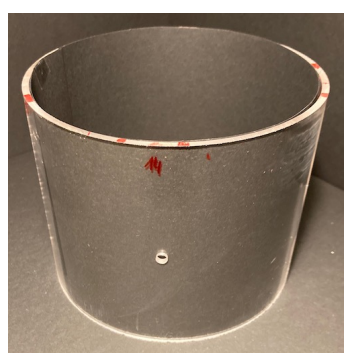

(h)

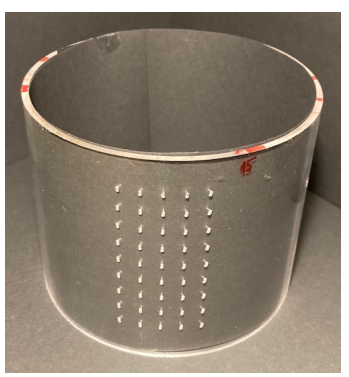

(i)

Fig. 4. Damages applied to the plastic cover of the OS1-64: (a) reference without damage (id:16), (b) multiple vertical scratches (id:10), (c) multiple stone scratches (id:11), (d) diagonal stone scratches (id:17), (e) one vertica crack (id:32), (f) one hole $\varnothing 1.4 \mathrm{~mm}$ (id:12), (g) one hole $\varnothing 2.5 \mathrm{~mm}$ (id:13), (h) one hole $\varnothing 5 \mathrm{~mm}$ (id:14), (i) multiple holes $\varnothing 1.4 \mathrm{~mm}$ (id:15). We attached black paper at the inside of the covers to increase the contrast of the images.

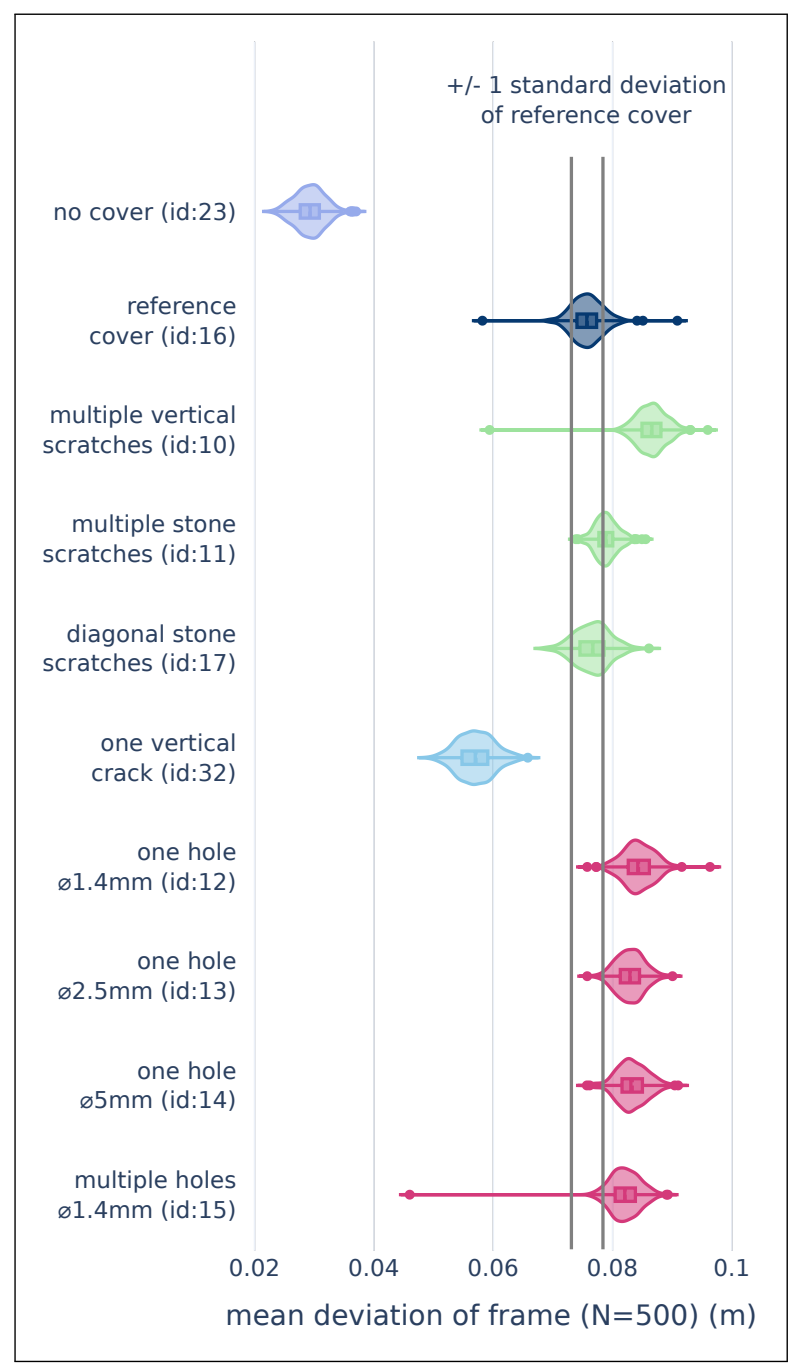

Fig. 5. Violin plots of deviation between OS1-64 points on the target and the fitted ideal plane. 500 point clouds per cover were considered. The vertical lines are placed at $+/-1$ standard deviation from the mean of the reference cover.

from the distribution of the reference cover (id:16). The results indicate that sensor cover damages have effects on point cloud data. Therefore, future work could focus on the development of appropriate fault detection algorithms. In a further step appropriate fault recovery and correction mechanisms may be developed to react on the detected fault. Furthermore, detected faults are an integral information for sensor fusion algorithms. A lidar sensor with a higher resolution than the OS1-64 can be used for better ground truth acquisition for further experiments. Moreover, the influence of sensor cover damages on beam divergence and correctness of angular measurements can be conducted in further research.

\section{ACKNOWLEDGMENT}

We would like to express our thanks to Oliver Mariani for his support in building the test setup. 


\section{REFERENCES}

[1] ERTRAC Working Group, "Connected automated driving roadmap," European Road Transport Research Advisory Council, Tech. Rep., 2017. [Online]. Available: https://www.ertrac.org/uploads/documentsearch/ id48/ERTRAC_Automated_Driving_2017.pdf

[2] —-, "Safe road transport roadmap, towards vision zero: Roads without victims," European Road Transport Research Advisory Council, Tech. Rep., 2019. [Online]. Available: https://www.ertrac.org/uploads/ documentsearch/id58/ERTRAC-Road-Safety-Roadmap-2019.pdf

[3] SAE On-road Automated Vehicle Standards Comittee, "Taxonomy and definitions for terms related to driving automation systems for on-road motor vehicles," std., SAE International, 2018. [Online]. Available: https://doi.org/10.4271/J3016_201806

[4] J. Shuttleworth, "Sae standards news: J3016 automated-driving graphic update," 2019. [Online]. Available: https://www.sae.org/news/2019/01/ sae-updates-j3016-automated-driving-graphic

[5] D. Watzenig and M. Horn, Eds., Automated Driving - Safer and More Efficient Future Driving. Springer, 2017. [Online]. Available: https://doi.org/10.1007/978-3-319-31895-0

[6] T. Goelles, B. Schlager, and S. Muckenhuber, "Fault detection, isolation, identification and recovery (fdiir) methods for automotive perception sensors including a detailed literature survey for lidar," MDPI Sensors, vol. 20(13), no. 3662, 2020. [Online]. Available: https://doi.org/10.3390/s20133662

[7] C. Goodin, D. Carruth, M. Doude, and C. Hudson, "Predicting the Influence of Rain on LIDAR in ADAS," Electronics, vol. 8(1), no. 89, 2019. [Online]. Available: https://doi.org/10.3390/electronics8010089

[8] R. Heinzler, P. Schindler, J. Seekircher, W. Ritter, and W. Stork, "Weather influence and classification with automotive lidar sensors," in IEEE Intelligent Vehicles Symposium (IV), Paris, F, June 9-12, 2019. IEEE, Piscataway, NJ, 2019, pp. 1527-1534. [Online]. Available: https://doi.org/10.1109/IVS.2019.8814205

[9] S. Hasirlioglu, I. Doric, A. Kamann, and A. Riener, "Reproducible Fog Simulation for Testing Automotive Surround Sensors," in 2017 IEEE 85th Vehicular Technology Conference (VTC Spring). Sydney, NSW: IEEE, 2017, pp. 1-7. [Online]. Available: https: //doi.org/10.1109/VTCSpring.2017.8108566

[10] S. Zang, M. Ding, D. Smith, P. Tyler, T. Rakotoarivelo, and M. A. Kaafar, "The Impact of Adverse Weather Conditions on Autonomous Vehicles: How Rain, Snow, Fog, and Hail Affect the Performance of a Self-Driving Car," IEEE Vehicular Technology Magazine, vol. 14, no. 2, pp. 103-111, 2019. [Online]. Available: https://doi.org/10.1109/MVT.2019.2892497

[11] M. Kutila, P. Pyykönen, H. Holzhüter, M. Colomb, and P. Duthon, "Automotive LiDAR performance verification in fog and rain," in 2018 21st International Conference on Intelligent Transportation Systems (ITSC). Maui, HI: IEEE, 2018, pp. 1695-1701. [Online]. Available: https://doi.org/10.1109/ITSC.2018.8569624

[12] M. Trierweiler, P. Caldelas, G. Gröninger, T. Peterseim, and C. Neumann, "Influence of sensor blockage on automotive lidar systems," in 2019 IEEE SENSORS, 2019, pp. 1-4.

[13] J. R. V. Rivero, I. Tahiraj, O. Schubert, C. Glassl, B. Buschardt, M. Berk, and J. Chen, "Characterization and simulation of the effect of road dirt on the performance of a laser scanner," in IEEE International Conference on Intelligent Transportation Systems (ITSC), Yokohama, Japan, Oct. 2017. [Online]. Available: https://doi.org/10.1109/ITSC.2017.8317784

[14] Ouster, Inc., "Ouster os1-64 (gen1)," data sheet, 2019.

[15] Stanford Artificial Intelligence Laboratory et al., "Robotic operating system," May 2018. [Online]. Available: www.ros.org

[16] S. Muckenhuber, H. Holzer, and Z. Bokaj, "Automotive lidar modelling approach based on material properties and lidar capabilities," MDPI Sensors, vol. 20(11), no. 3309, 2020. [Online]. Available: https://doi.org/10.3390/s20113309

[17] Ingemann Components, "I-reflect 0.23 with and without adhesive," data sheet. [Online]. Available: https://www.ingemanncomponents.com/wp-content/uploads/2018/ 06/I-Reflect-0.23-with-and-without-adhesive.pdf

[18] 3M, "Product bulletin: $3 \mathrm{~m}^{\mathrm{TM}}$ scotchcal $^{\mathrm{TM}}$ light management film 3635-100," data sheet, 2020. [Online]. Available: https://multimedia.3m.com/mws/media/50650O/ 3m-light-enhancement-film-3635-100-product-bulletin.pdf
[19] —, "Reflektierende folien $3 \mathrm{~m}^{\mathrm{TM}}$ engineer grade prismatic serie 3430," data sheet, 2020. [Online]. Available: https://multimedia.3m.com/mws/media/845099O/ technical-information-3m-engineer-grade-prismatic-series-3430.pdf

[20] SphereOptics GmbH, "Zenith lite ${ }^{\mathrm{TM}}$ targets," data sheet, 2015. [Online]. Available: https://sphereoptics.de/en/wp-content/uploads/sites/ 3/2014/03/Zenith-Lite-Targets-E.pdf

[21] Q.-Y. Zhou, J. Park, and V. Koltun, "Open3D: A modern library for 3D data processing," 2018. [Online]. Available: https://arxiv.org/abs/1801. 09847 